\title{
Neumonía asociada a cuidados
} de la salud: revisión de la evidencia publicada

\author{
Recibido: 05/06/17 Aceptado: 04/07/17
}

Lautaro de Vedia' ${ }^{1}$ Eugenia Di Líbero², Pablo Scapellato³, Javier Desse ${ }^{4}$.

\section{RESUMEN}

El concepto de neumonía asociada a cuidados de la salud (NACS) surgió a partir de la presunción de que los patógenos causantes del cuadro tendrían mayores probabilidades de ser microorganismos multiresistentes (MOMR), por lo que el esquema de tratamiento antibiótico debía ser diferente al requerido en neumonía adquirida en la comunidad (NAC). Sin embargo, la evidencia que sustenta esa idea no es lo suficientemente robusta. Dado lo complejo del tema, y su elevado impacto en el consumo exagerado de antibióticos, se presenta esta revisión. Es posible que la ausencia de los factores de riesgo usualmente descriptos, o la presencia de solo uno, sugiera poca probabilidad de MOMR, por lo que el abordaje terapéutico debería ser similar al de NAC. Por el contrario, ante la acumulación de factores de riesgo o frente a cuadros severos se podría considerar la cobertura de MOMR mediante un esquema de espectro ampliado.

Palabras clave: neumonía asociada a cuidados de la salud, microorganismos multiresistentes, neumonía.
'Unidad de Asistencia Respiratoria, Departamento de Terapia Intensiva, Hospital Francisco J. Muñiz, CABA. Sociedad Argentina de Infectología (Comisión de Uso Adecuado de Recursos).

${ }^{2}$ Hospital Interzonal General de Agudos "Evita", Lanús, Provincia de Buenos Aires. Sociedad Argentina de Infectología (Comisión de Uso Adecuado de Recursos).

3Unidad de Infectología, Hospital D. F. Santojanni, CABA. Sociedad Argentina de Infectología (Comisión de Uso Adecuado de Recursos).

${ }^{4}$ Hospital Zonal General de Agudos "Dr. Enrique Erill", Belén de Escobar, Provincia de Buenos Aires. Sociedad Argentina de Infectología (Comisión de Uso Adecuado de Recursos).

Dirección para correspondencia: Lautaro de Vedia. Uspallata 2272, CABA. lautarodevedia@gmail.com

Los autores manifiestan la inexistencia de algún tipo de conflicto de intereses. 


\section{Introducción}

Dadolocomplejodeltema,ysuelevadoimpactoenelconsumo exagerado de antibióticos, tal vez injustificado en muchos La definición de neumonía asociada a cuidados de la salud casos, creemos necesario presentar esta revisión. De todos (NACS) surgió de las Guías para el manejo de la Neumonía modos, es fundamental incrementar los esfuerzos dirigidos Intrahospitalaria 2005 IDSA-ATS (1). La idea que justificaba a la investigación de la distribución de microorganismos la existencia de esta nueva categoría se basaba en la vinculados con NACS y su patrón de susceptibilidad, así presunción de que los patógenos causantes de neumonía como de los factores de riesgo intervinientes.

en los grupos de pacientes considerados como "asociados a cuidados de la salud" tendrían mayores probabilidades de ser microorganismos multiresistentes (MOMR) por lo que esquema de tratamiento antibiótico debía ser necesariamente diferente al requerido en pacientes con neumonía adquirida en la comunidad (NAC) (2).

En esas guías se afirmaba que los factores de riesgo para NACS eran: hospitalización de dos o más días en los 90 días previos, residencia en geriátricos o en unidades de cuidados crónicos, terapia de infusión endovenosa domiciliaria o en hospital de día, diálisis crónica en los últimos 30 días requerimiento de cuidados de heridas en domicilio y tener a un miembro de la familia con un patógeno multiresistente. También se mencionaba que en el caso de sospecharse NACS se debía evaluar si existían criterios que hicieran sospechar la presencia de MOMR. En caso de que no, el tratamiento antibiótico debe ser de espectro limitado. Por el contario, en situaciones de factores sugerentes de MOMR, la terapia antibiótica debía ser de amplio espectro.

Esta postura fue avalada por un articulo publicado por Kollef en 2005 (3) y aceptada en una encuesta realizada a un panel de once expertos y a 744 miembros de la IDSA (4). Sin embargo, más de la mitad de los que respondieron la encuesta (tanto expertos como miembros de IDSA) aceptó la postura de considerar a la NACS como una entidad separada pero con ciertas reservas.

Posteriormente se publicaron muchos trabajos sobre el tema. Los resultados no fueron lo suficientemente contundentes como para sostener la necesidad de mantene esa categoría en la clasificación $(5,6,7,8)$. Finalmente, en la reciente actualización de dichas guías, publicadas en 2016, el concepto de NACS fue reconsiderado y se resolvio excluirlo de las recomendaciones, debido a que la idea de que los pacientes relacionados con el sistema de salud tuvieran mayor riesgo de MOMR fue ampliamente cuestionada (9). Por el contrario, muchos expertos consideran que son las características clínicas subyacentes de estos pacientes las que constituyen el verdadero riesgo para MOMR (10).

\section{Estudios basados en la epidemología}

Diferentes estudios intentaron encontrar factores epidemiológicos que vincularan a la NACS con una mayor frecuencia de MOMR. Sin embargo, los resultados no lograron demostrar fehacientemente esa relación (Tabla 1).

En un estudio epidemiológico realizado en el Reino Unido se compararon las características de 277 pacientes con NACS con un grupo control de 1071 sujetos con NAC (11). El estudio mostró que la NACS se asoció con mayor mortalidad en comparación con NAC. Sin embargo, la población con NACS tenía mayor promedio de edad (76+65-83 años vs 65+48-77 años, $p<0,0001$ y mayor proporción de insuficiencia cardíaca ( $29,6 \%$ vs $15,8 \%, p<0,0001)$, EPOC ( $30 \%$ vs $18,3 \%, p<0,0001$ ) y enfermedad cerebrovascular $(18,8 \%$ vs $10,1 \%, p=0,0001)$, por lo que no es claro si la diferente mortalidad obedece a la presencia de comorbilidades o a la existencia de distintos patógenos. El estudio resulta insuficiente como para sostener necesidad de cambios en los tratamientos empíricos.

Un estudio retrospectivo que analizó una gran base de datos en Estados Unidos, entre 2010 y 2011 tuvo como objetivo determinar la tasa de patógenos MOMR en pacientes con NAC y establecer si NACS es un factor de riesgo para MOMR (12). Los resultados mostraron que sobre 521 pacientes (50,5\% NAC y $49,5 \%$ NACS), en los que la edad promedio fue de 65 años, solo se registraron 20 aislamientos de MOMR (3,8\% en total, $5,9 \%$ en NACS y $1,9 \%$ en NAC). Sin embargo, no pudo demostrarse que NACS fuera un factor de riesgo para MOMR (OR 1,95, IC 95\% 0,66-5,80, $\mathrm{p}=0,23$ ). La mortalidad fue superior en NACS $(5,8 \%)$ en comparación con NAC $(0,4 \%)$. Los factores de riesgo para MOMR fueron infección colonización por Pseudomona aeruginosa en el último año (5 pacientes) (OR 7,43, IC 95\% 2,24-24,61), haber recibido ATB en los últimos 90 días (12 pacientes) (OR 2,90, IC 95\% 1,13$7,45)$ y tener enfermería domiciliaria (13 pacientes) (OR 4,19, IC $95 \%(1.55-11.31)$
Tabla 1. Estudios basados en la epidemiología

\begin{tabular}{|c|c|c|c|}
\hline Autor & $\begin{array}{l}\text { País y número } \\
\text { de pacientes }\end{array}$ & Resultados & Conclusión \\
\hline $\begin{array}{l}\text { Chalmers JD et al. } \\
\text { Clin Infect Dis. 2011; } \\
\text { 53(2):107-13 (11) }\end{array}$ & $\begin{array}{l}\text { Reino Unido } \\
\text { Estudio prospectivo } \\
\text { observacional } \\
277 \text { pacientes con NACS y } \\
1071 \text { con NAC }\end{array}$ & $\begin{array}{l}\text { NACS se asoció con mayor } \\
\text { mortalidad, pero esto } \\
\text { pareciera relacionarse más } \\
\text { con las comorbilidades, ya } \\
\text { que la población con NACS } \\
\text { tenía mayor promedio de } \\
\text { edad y mayor proporción de } \\
\text { comorbilidades (IC, EPOC, } \\
\text { enfermedad cerebro vascular). }\end{array}$ & $\begin{array}{l}\text { El estudio no confirma la } \\
\text { necesidad de cambios en los } \\
\text { tratamientos empíricos. }\end{array}$ \\
\hline $\begin{array}{l}\text { Gross AE et al. Antimicrob } \\
\text { Agents Chemother. 2014; } \\
\text { 58(9):262-8 (12) }\end{array}$ & $\begin{array}{l}\text { Estados Unidos } \\
\text { Estudio retrospectivo } \\
521 \text { pacientes, } \\
261(50,5 \%) \text { con NAC y } 260 \\
(49,5 \%) \text { con NACS }\end{array}$ & $\begin{array}{l}\text { Solo se registraron } 20 \\
\text { aislamientos de MOMR (3,8\%). } \\
\text { La tasa de MOMRR fue de } 5,9 \% \\
\text { en NACS y } 1,9 \% \text { en NAC. } \\
\text { No pudo demostrarse que } \\
\text { NACS fuera un factor de riesgo } \\
\text { para MOMR (RR 1,95, IC } 95 \% \\
0,66-5,80, p=0,23) \text {. } \\
\text { La mortalidad fue superior en } \\
\text { NACS (5,8\%) en comparación } \\
\text { con NAC }(0,4 \%) \text {. }\end{array}$ & $\begin{array}{l}\text { Los factores de riesgo para } \\
\text { MOMR fueron infección } \\
\text { o colonización por } P \text {. } \\
\text { aeruginosa en el último } \\
\text { año, haber recibido ATB en } \\
\text { los últimos } 90 \text { días y tener } \\
\text { enfermería domiciliaria. }\end{array}$ \\
\hline $\begin{array}{l}\text { Ceccato A et al. Medicina. } \\
\text { 2014;74:19-23 } \\
\text { (13) }\end{array}$ & $\begin{array}{l}\text { Argentina } \\
\text { Estudio prospectivo } \\
\text { observacional } \\
60 \text { pacientes con NACS }\end{array}$ & $\begin{array}{l}\text { Solo se consiguió realizar } \\
\text { aislamiento microbiológico en } \\
18 \text { pacientes ( } 30 \% \text { ), siendo el } \\
\text { más frecuente } S \text {. pneumoniae } \\
\text { ( } 9 \text { casos). } \\
\text { El } 77 \% \text { de los pacientes tenía } \\
\text { un PSI elevado (Clase } 4 \text { o } 5) \\
\text { sugiriendo que se trataba de } \\
\text { una población de alto riesgo, } \\
\text { independientemente de los } \\
\text { patógenos involucrados. }\end{array}$ & $\begin{array}{l}\text { No se justifican cambios } \\
\text { en el esquema antibiótico } \\
\text { utilizado habitualmente. }\end{array}$ \\
\hline $\begin{array}{l}\text { Vallés J et al. Intensive Care } \\
\text { Med. 2014; 40(4):572-81 (14) }\end{array}$ & $\begin{array}{l}\text { España } \\
133 \text { con NAC y } 144 \text { con NACS }\end{array}$ & $\begin{array}{l}\text { Los pacientes con NACS } \\
\text { tuvieron más comorbilidades. } \\
\text { S. pneumoniae fue el patógeno } \\
\text { más frecuente: } 19,5 \text { en NACS y } \\
34,2 \% \text { en NAC ( } 0=0.001) \text {. } \\
\text { La incidencia de } P \text {. aeruginosa } \\
\text { y SAMR fue mayor en NACS } \\
\text { pero con muy baja frecuencia } \\
\text { en general. } \\
\text { La incidencia de MOMR fue de } \\
9,7 \% \text { en el grupo de NACS y de } \\
3,4 \% \text { en NAC ( } p=0.001) \text {. } \\
\text { La tasa de mortalidad no } \\
\text { difiríó NAC } 18,4 \%, N A C S \\
21,2 \% \text {. }\end{array}$ & $\begin{array}{l}\text { Un tratamiento de acuerdo } \\
\text { con las guías de NAC } \\
\text { sería útil en el } 90 \% \text { de los } \\
\text { pacientes con NACS. }\end{array}$ \\
\hline
\end{tabular}


En un trabajo observacional prospectivo realizado en nuestro país por profesionales del Hospital Posadas, en el que se incluyeron 60 casos de NACS, no se rescataron gérmenes

resistentes ni se constató una mortalidad elevada que Enlostrabajosquesepresentana continuaciónyse muestran justificara un cambio en el esquema antibiótico utilizado en la Tabla 2 fueron comparados diferentes abordajes habitualmente (13). El estudio fue pequeño y sólo en el 30\% terapéuticos en pacientes con NACS. de los episodios se alcanzó un diagnóstico bacteriológico. La población estudiada tenía una media de edad de 63 años En un trabajo que se llevó a cabo en los Estados Unidos fueron y si bien el 77\% de los pacientes tenía un PSI y un CURB-65 evaluados en forma retrospectiva 228 pacientes con NACS elevados, la mortalidad fue del $5 \%$

Un estudio prospectivo observacional llevado a cabo en 34 UCI de España evaluó 726 casos de neumonía (14). De ellas, 449 eran NAC, 133 NACS y 144 neumonías en pacientes inmunocomprometidos. Los pacientes con NACS tuvieron más comorbilidades que aquellos con NAC y requirieron en mayor proporción ventilación mecánica. La tasa de rescate microbiológico fue cercana al 50\% y si bien Streptococcus pneumoniae fue el patógeno más frecuente, la proporción fue muy diferente: 19,5 en NACS y $34,2 \%$ en NAC ( $p=0.001)$. La incidencia de $P$. aeruginosa y Staphylococcus aureus meticilino resistente (SAMR) fue mayor en el grupo de NACS ( $3 \%$ vs $1,6 \%$ y $2,3 \%$ vs $0,2 \%$ respectivamente), pero igualmente muy baja en general. La incidencia de MOMR fue de $9,7 \%$ en el grupo de NACS y de $3,4 \%$ en el de NAC ( $p=0.001)$. La tasa de mortalidad no difirió entre NAC y NACS: $18,4 \%$ vs $21,2 \%$. En e análisis de regresión logística, el factor que más fuertemente se asoció con MOMR fue NACS (OR 2,79, IC 95\% 1,27-6,12). No obstante, los autores señalaron que un tratamiento de acuerdo con las guías pacientes con NACS.

evaluados en forma retrospectiva 228 pacientes con NACS fueron similares en cuanto a severidad del cuadro, datos

\section{Estudios basados en la evaluación de} tratamientos admitidos entre 2005 y 2010 (15). Se excluyeron los pacientes que ingresaron a UTI o que tenían neumonía intrahospitalaria. El análisis se basó en la pauta de tratamiento elegido por los médicos tratantes: si la terapia empírica fue determinada según guías de NACS ( $n=106)$ (inclusión de un agente anti- $P$. aeruginosa) o según guías de NAC ( $n=122)$. Ambos grupos demográficos, factores de riesgo y comorbilidades. Si bien la tasa de cura fue similar en los dos grupos, tanto los días de terapia endovenosa como los de internación fueron significativamente mayores en el grupo tratado con pauta de NACS.

En un estudio de cohorte basado en la población, llevado a cabo en más de 150 hospitales de Estados Unidos, se incluyeron un total de 15.071 pacientes con, al menos, un criterio de NACS y que hubieran recibido tratamiento antibiótico en las primeras 48 horas del ingreso hospitalario (16). Fueron excluidos los sujetos que ingresaban a UTI. Los tratamientos fueron divididos según fueran concordantes con las guías de NAC $(75,7 \%)$, concordantes con las guías de NACS $(8 \%)$ o no concordantes con ninguna guía $(16,3 \%)$. Los resultados mostraron que la mortalidad a 30 y 90 fue significativamente más elevada entre los pacientes que recibieron un tratamiento concordante con guía de NACS ( $22,8 \%$ y $37,8 \%$ respectivamente) que entre aquellos que fueron tratados de forma concordante con guías de NAC (9,9\% y 19,8\% respectivamente). Los más fuertes predictores de mortalidad a 30 días fueron la admisión hospitalaria reciente (OR 2,49, IC 95\% 2,12-2,94) y la terapia concordante con guía de NACS (OR 2,18, IC 95\% 1,86-2,55). Los autores consideraron improbable que la mayor mortalidad observada en el grupo NACS se debiera al tratamiento, pudiendo existir confundidores no captados por el diseño que hayan influenciado la diferencia en los resultados (severidad de la enfermedad, status funcional, etc.)

\section{Tabla 2. Estudios basados en la evaluación de tratamientos}

\begin{tabular}{|c|c|c|c|}
\hline Autor & $\begin{array}{l}\text { País y número } \\
\text { de pacientes }\end{array}$ & Resultados & Conclusión \\
\hline $\begin{array}{l}\text { Chen Jl et al. Ann } \\
\text { Pharmacother. 2013; 47:9-19 } \\
\text { (15) }\end{array}$ & $\begin{array}{l}\text { Estados Unidos } \\
\text { Estudio retrospectivo } \\
228 \text { pacientes con NACS, } 106 \\
\text { tratados con pauta de NACS } \\
\text { (inclusión de un agente anti-P. } \\
\text { aeruginosa) y } 122 \text { tratados } \\
\text { con pauta de NAC }\end{array}$ & $\begin{array}{l}\text { Ambos grupos fueron } \\
\text { similares en cuanto a datos } \\
\text { demográficos y severidad del } \\
\text { cuadro. } \\
\text { Los pacientes tratados con } \\
\text { pautas de NACS tuvieron } \\
\text { similar tasa de cura clínica } \\
\text { global pero más días de } \\
\text { terapia endovenosa y más días } \\
\text { de internación. }\end{array}$ & $\begin{array}{l}\text { En pacientes con NACS los } \\
\text { tratamientos con regímenes } \\
\text { de antibióticos que } \\
\text { incluyeron cobertura anti-P. } \\
\text { aeruginosa no incrementaron } \\
\text { las tasas de cura clínica } \\
\text { y se asociaron con mayor } \\
\text { cantidad de días de terapia. }\end{array}$ \\
\hline $\begin{array}{l}\text { Attridge RT et al. Eur Respir J. } \\
\text { 2011; 38:878-887 (16) }\end{array}$ & $\begin{array}{l}\text { Estados Unidos } \\
\text { Estudio de cohorte } \\
\text { retrospectivo } \\
15.071 \text { pacientes, } \\
75,7 \% \text { recibieron tratamiento } \\
\text { concordante con guías de } \\
\text { NAC, 8\% con guias de NACS } \\
\text { y } 16,3 \% \text { no concordante con } \\
\text { ninguna guía }\end{array}$ & $\begin{array}{l}\text { La mortalidad a } 30 \text { y } 90 \\
\text { fue significativamente más } \\
\text { elevada entre los pacientes } \\
\text { que recibieron un tratamiento } \\
\text { concordante con guía de } \\
\text { NACS. } \\
\text { Los más fuertes predictores de } \\
\text { mortalidad a } 30 \text { días fueron la } \\
\text { admisión hospitalaria reciente } \\
\text { y la terapia concordante con } \\
\text { guía de NACS. }\end{array}$ & $\begin{array}{l}\text { En pacientes no graves, la } \\
\text { terapia acorde con las guias } \\
\text { de NACS no se asoció con } \\
\text { mejoría en la sobrevida, en } \\
\text { comparación con la terapia } \\
\text { acorde con las guías de NAC. }\end{array}$ \\
\hline
\end{tabular}

\section{Estudios basados en}

\section{la estratificación del riesgo}

Algunos estudios abordaron el análisis del riesgo de tener una neumonía por MOMR en pacientes con criterios de NACS, intentando estratificarlo y predecir su diagnóstico (Tabla 3).

En un análisis retrospectivo llevado en Estados Unidos sobre una población de 977 pacientes con NAC (17) se encontraron entre las etiologías SAMR (22,3\% de los casos), P. aeruginosa $(19,1 \%)$ y $S$. pneumoniae $(19,1 \%)$. Se estableció un score que intentaba predecir la infección por MOMR, asignando puntaje en base a los antecedentes de contacto con el sistema de salud, la condición clínica actual y las comorbilidades. E estudio mostró que los casos en los que existió el aislamiento de algún MOMR el score promedio fue significativamente mayor.

Un estudio observacional, prospectivo, realizado en Italia, incluyó 935 episodios de neumonía hospitalizados entre 2008 y 2010, ensayando otro score, el cual ponderó de modo distinto a los diferentes antecedentes y condiciones clínicas, agregando la consideración acerca de otras comorbilidades, así como la toma de antimicrobianos previa. Este score mostró una sensibilidad de $78 \%$ y una especificidad de $56 \%$ para predecir la presencia de MOMR (18)

tro estudio retrospectivo observacional realizado en Taiwán enroló 530 pacientes con NACS, con el objetivo de investigar si el PSI podría ser un método útil para predecir la presencia de MOMR y categorizar así el riesgo del episodio (19). Un (206/530) presentó aislamientos de MOMR, entre los cuales $P$. aeruginosa fue el más prevalente (24\%). La frecuencia de MOMR aumentó en la medida que se incrementaba el PSI. De todos modos, se trata de un estudio realizado en un único centro, en el que hay una incidencia excesivamente alta de $P$. aeruginosa.

Finalmente, otro trabajo llevado a cabo en España (20) y etiológico evaluó la utilidad de un score clínico-epidemiológico que intentara establecer el riesgo de presentar un MOMR. El score intenta establecer el incremento en el riesgo de presentar un patógeno resistente (Grupo PES: $P$. aeruginosa, Enterobacteriaceae BLEE +, SAMR) en pacientes con NAC. En el estudio no fueron incluidos pacientes residentes en instituciones de cuidados crónicos. Los resultados mostraron que un score igual o mayor a 5 puntos se asoció con riesgo que incluyó 1597 pacientes con neumonía con diagnóstico 
incrementado de MOMR. Debe destacarse que solo en un desarrollado por Niederman (22), en el cual se consideraban $6 \%$ de los casos de neumonía se identificó un MOMR: estos la severidad del cuadro (necesidad de ventilación mecánica pacientes fueron significativamente mayores, habian recibido o admisión en UTI) y la presencia de factores de riesgo para antibióticos previos con mayor frecuencia, presentaron más MOMR (inmunosupresión, hospitalización en los últimos frecuentemente fallo renal agudo y sufrieron una mayor 90 días, pobre status funcional, antibiótico terapia en los 6 mortalidad a 30 días. Finalmente, el grupo PES constituyó meses previos). El score asignaba un punto por cada una de una pequeña proporción del total de neumonías, pero se esas variables. En los sujetos con un score de 0-1 se indicaba asoció con mayor mortalidad, por lo que, según la conclusión una pauta de tratamiento de NAC, mientras que con un score a la que arribaron los autores, aquellos pacientes con un de 2 o más se indicaba una antibioticoterapia ampliada. mayor score de riesgo podrían beneficiarse con un esquema Si bien la mortalidad en los pacientes con NACS fue antibiótico diferente al de la NAC. significativamente mayor $(13,7 \%$ vs $5,6 \%, \mathrm{p}=0.017)$, solo un $7 \%$ de los pacientes con NACS recibió una terapia antibiótica En un estudio más reciente que se llevó a cabo en Japón (21) inapropiada, lo que sugiere que fue mayor el impacto de los se intentó validar de manera prospectiva un score de riesgo factores asociados con el paciente que los vinculados con la terapia antibiótica.

\section{Tabla 3. Estudios basados en la estratificación del riesgo}

\begin{tabular}{|c|c|c|c|}
\hline Autor & $\begin{array}{l}\text { País y número } \\
\text { de pacientes }\end{array}$ & Resultados & Conclusión \\
\hline $\begin{array}{l}\text { Shorr AF et al. Clin Infect Dis. } \\
\text { 2012; 54(2):193-198 (17) }\end{array}$ & $\begin{array}{l}\text { Estados Unidos } \\
\text { Análisis retrospectivo } \\
977 \text { pacientes con NAC }\end{array}$ & $\begin{array}{l}\text { Se estableció un sistema de } \\
\text { score, asignando } 4 \text { puntos } \\
\text { cuando había hospitalización } \\
\text { reciente, } 3 \text { cuando había } \\
\text { residencia en unidades de } \\
\text { cuidados crónicos, } 2 \text { para } \\
\text { la presencia de enfermedad } \\
\text { card́acac crónica y } 1 \text { cuando se } \\
\text { registraba ingreso a UTI dentro } \\
\text { de las } 24 \text { hs de evaluación en } \\
\text { la guardia. }\end{array}$ & $\begin{array}{l}\text { Los casos en los que había } \\
\text { MOMR tuvieron un score } \\
\text { promedio significativamente } \\
\text { mayor ( } 4 \text { vs } 1, p>0.01) \text {. }\end{array}$ \\
\hline $\begin{array}{l}\text { Aliberti S et al. } \\
\text { Clin Infect Dis. 2012; } \\
\text { 54(4):470-478 (18) }\end{array}$ & $\begin{array}{l}\text { Italia } \\
\text { Estudio observacional } \\
\text { prospectivo } \\
935 \text { NACS }\end{array}$ & $\begin{array}{l}\text { En este estudio se asignó un } \\
\text { puntaje de } 5 \text { para la presencia } \\
\text { de fallo renal crónico, } 4 \text { para } \\
\text { hospitalización durante } \\
\text { dos o más días en los } 90 \\
\text { días precedentes, } 3 \text { para la } \\
\text { residencia de unidades de } \\
\text { cuidados crónicos y } 0,5 \text { para } \\
\text { la presencia de uno o más de } \\
\text { los siguientes: diabetes, EPOC, } \\
\text { enfermedad cerebrovascular, } \\
\text { antibióticos en los } 90 \text { días } \\
\text { previos, inmunosupresión, } \\
\text { cuidados domiciliarios de } \\
\text { herida o terapia de infusión } \\
\text { domiciliaria. }\end{array}$ & $\begin{array}{l}\text { El abordaje según este score } \\
\text { mostró una sensibilidad del } \\
78 \% \text { y una especificidad del } \\
56 \% \text {. }\end{array}$ \\
\hline $\begin{array}{l}\text { Wang PH et al. J Formos Med } \\
\text { Assoc. } 2016 \text { May; } 115(5): 356- \\
63 \text { (19) }\end{array}$ & $\begin{array}{l}\text { Taiwán } \\
\text { Estudio retrospectivo } \\
\text { observacional } \\
530 \text { pacientes con NACS }\end{array}$ & $\begin{array}{l}\text { El objetivo fue investigar si el } \\
\text { PSI poría ser un método útil } \\
\text { para predecir la presencia de } \\
\text { MOMR y categorizar el riesgo } \\
\text { del episodio. }\end{array}$ & $\begin{array}{l}\text { La frecuencia de MOMR } \\
\text { aumentó en la medida que se } \\
\text { incrementaba el PSI. }\end{array}$ \\
\hline
\end{tabular}

\begin{tabular}{|c|c|c|c|}
\hline Autor & $\begin{array}{l}\text { País y número } \\
\text { de pacientes }\end{array}$ & Resultados & Conclusión \\
\hline $\begin{array}{l}\text { Prina E et al. } \\
\text { Ann Am Thorac Soc. 2015; } \\
\text { 12(2):153-60 (20). }\end{array}$ & $\begin{array}{l}\text { España } \\
\text { Estudio prospectivo } \\
\text { observacional } \\
1597 \text { pacientes con neumonía }\end{array}$ & $\begin{array}{l}\text { Se evaluó el incremento } \\
\text { en el riesgo de presentar } \\
\text { un patógeno resistente } \\
\text { (Grupo PES: P. aeruginosa, } \\
\text { Enterobacteriaceae BLEE +, } \\
\text { SAMR). } \\
\text { Los factores de riesgo que } \\
\text { conformaban este score } \\
\text { fueron la edad mayor a } 40 \\
\text { y } 65 \text { años, el uso previo de } \\
\text { antibióticos, los trastornos } \\
\text { respiratorios crónicos, la } \\
\text { enfermedad renal crónica y el } \\
\text { deterioro de la conciencia. } \\
\text { Un score igual o mayor a } 5 \\
\text { puntos se asoció con riesgo } \\
\text { incrementado para PES. } \\
\text { El grupo PES constituyo una } \\
\text { pequeña proporción del total } \\
\text { de neumonías (6\%), pero se } \\
\text { asoció con mayor mortalidad. }\end{array}$ & $\begin{array}{l}\text { Aquellos pacientes con } \\
\text { un mayor score de riesgo } \\
\text { podrían beneficiarse con } \\
\text { un esquema antibiótico } \\
\text { diferente al de la NAC. }\end{array}$ \\
\hline $\begin{array}{l}\text { Maruyama Tet al. Clin Infect } \\
\text { Dis 2013; 57(10):1373-83 } \\
\text { (21). }\end{array}$ & $\begin{array}{l}\text { Japón } \\
\text { Estudio prospectivo, con } \\
\text { intervención } \\
445 \text { pacientes, } 321 \text { con NACS } \\
\text { y } 124 \text { con NAC }\end{array}$ & $\begin{array}{l}\text { El score consideraba un } \\
\text { punto por cada una de las } \\
\text { siguientes variables: severidad } \\
\text { del cuadro (necesidad de } \\
\text { ventilación mecánica o } \\
\text { admisión en UTI) y presencia } \\
\text { de factores de riesgo para } \\
\text { MOMR (inmunosupresión, } \\
\text { hospitalización en los últimos } \\
90 \text { días, pobre status funcional, } \\
\text { antibiótico terapia en los } 6 \\
\text { meses previos). } \\
\text { En los sujetos con un score } \\
\text { de } 0-1 \text { se indicaba una pauta } \\
\text { de tratamiento de NAC, } \\
\text { mientras que con un score } \\
\text { de } 2 \text { o más se indicaba una } \\
\text { antibioticoterapia ampliada. }\end{array}$ & $\begin{array}{l}\text { Si bien la mortalidad en los } \\
\text { pacientes con NACS fue } \\
\text { significativamente mayor } \\
\text { ( } 13,7 \% \text { vs } 5,6 \%, p=0.017), \\
\text { sólo un } 7 \% \text { de los pacientes } \\
\text { con NACS recibió una terapia } \\
\text { antibiótica inapropiada. }\end{array}$ \\
\hline
\end{tabular}




\section{Conclusiones}

Los datos son muy variables, tal como sugiere una revisión En conclusión, parece injustificado el uso rutinario de trabajos sobre NACS y se observó que los criterios utilizados presenten algún factor de riesgo para NACS. Es posible que la para definir la entidad son diferentes en todos: nueve de los ausencia de cualquiera de los factores de riesgo usualmente diez trabajos consideraron a la residencia en geriátricos como descriptos, o la presencia de solo uno de ellos, sugiera poca un factor de riesgo para NACS, pero solo cinco incluyeron probabilidad de MOMR, por lo que el abordaje terapéutico entre esos factores de riesgo al ingreso hospitalario en los podría ser similar al de un caso de NAC. Por el contrario, ante 90 días previos, a la terapia de infusión domiciliaria o a la la acumulación de factores de riesgo o frente a situaciones hemodílisis crónca Por orta pate las tasas de rescate en las que la sevidad del cuadro y/o las comorilidades microbiológico en la mayoría de los estudios fue muy baja: concomitantes disminuyan la capacidad del paciente de $9,2 \%$ en el estudio de Attridge (16) y $25 \%$ en el de Chen (15). tolerar un fallo en el esquema empírico inicial, se podría Finalmente, la distribución de microorganismos identificados considerar necesaria la cobertura de MOMR mediante un varía mucho de un estudio a otro: SAMR fue aislado en el esquema de espectro ampliado. De todos modos, todas estas $2,4 \%$ de los casos en un estudio (22) y en el $22 \%$ (17) en otro, sugerencias son absolutamente provisorias y sustentadas mientras que $P$. aeruginosa osciló entre $1,6 \%(23)$ y $24 \%(18)$. por muy escasa las avalen. Lo único indiscutible es la importancia que tiene la toma de muestras respiratorias para cultivo en los pacientes que se internan con NACS, a fin de contar con la posibilidad de descalonar el esquema antimicrobiano empírico inicial de ser posible. Es indudable que deben realizarse estudios clínicos a nivel local con el objetivo de contar con datos para orientar eventuales recomendaciones.

a fin de disminuir el uso innecesario de antibióticos (24). Se incluyeron nueve estudios clínicos y se concluyó en primer lugar que no todos los trabajos consideraban los mismos factores de riesgo. Los más importante fue que si bien algunos factores de riesgo mostraron una buena sensibilidad para detectar la probabilidad de asociación con presencia de MOMR (hospitalización en los últimos 90 días, uso de antibióticos en los 180 días previos, hemodiálisis), todos fallaron en términos de especificidad.

\section{Referencias}

1. American Thoracic Society; Infectious Diseases Society of America. Guidelines for the management of adults with hospital-acquired, ventilator-associated, and healthcare associated pneumonia. Am J Respir Crit Care Med. 2005 Feb 15; 171(4):388-416.

2. Friedman ND, Kaye KS, Stout JE, McGarry SA, Trivette $\mathrm{SL}$, Briggs JP, et al. Health care-associated bloodstream infections in adults: a reason to change the accepted definition of community-acquired infections. Ann Intern Med. 2002 Nov 19; 137(10):791-7.

3. Kollef MH, Shorr A, Tabak YP, Gupta V, Liu LZ, Johannes RS Epidemiology and outcomes of health-care-associated pneumonia: results from a large US database of culturepositive pneumonia. Chest. 2005 Dec;128(6):3854-62 Erratum in: Chest. 2006 Mar; 129(3):831.

4. Kollef $M H$, Morrow $L E$, Baughman RP, Craven $D E$ McGowan JE Jr, Micek ST, et al. Health care-associated pneumonia (HCAP): a critical appraisal to improve identification, management, and outcomes-proceedings of the HCAP Summit. Clin Infect Dis. 2008; 46 Supp 4:S296-334.

5. Attridge RT, Frei $\mathrm{CR}$. Health Care-associated Pneumonia: An Evidence-base Review. Am J Med. 2011; 124:689-97.

6. García-Vidal C, Viasus D, Roset A, Adamuz J, Verdaguer $\mathrm{R}$, Dorca J, et al. Low incidence of multidrug-resistan organisms in patients with healthcare-associated pneumonia requiring hospitalization. Clin Microbio Infect. 2011 Nov; 17(11):1659-65

7. Seymann GB, DiFrancesco $L$ Sharpe $B$, et al. The HCAP gap: differences between self-reported practice patterns and published guidelines for health care-associated pneumonia. Clin Infect Dis 2009; 49:1868-74.

8. Kett DH, Cano E, Quartin AA, et al. Improving medicine through pathway assessment of critical therapy of hospitalacquired pneumonia (IMPACTHAP) of hospitalacquired pneumonia (MPACT-HAP) investigators. Implementation of guidelines for management of possible multidrug-resistant pneumonia in intensive care: an observational,

9. Kalil AC, Metersky ML, Klompas M, Muscedere J, Sweeney DA, Palmer LB, et al. Management of Adults With Hospitalacquired and Ventilator-associated Pneumonia: 2016 Clinical Practice Guidelines by the Infectious Diseases Society of America and the American Thoracic Society Clin Infect Dis. 2016 Sep 1; 63(5):e61-e111.

10. Gross AE, Van Schooneveld TC, Olsen KM, Rupp ME, Bui $T H$ Forsung $E$ et al. Epidemiology and predictors of multidrug-resistant community-acquired and health care- associated pneumonia. Antimicrob Agents Chemother. 2014 Sep; 58(9):5262-8.

11. Chalmers JD, Taylor JK, Singnayan A. Fleming GB, Akrain AR, Mandel P et al. Epidemiology, Antibiotic Therapy, and Clinical Outcomes in Health Care-Associated Pneumonia: A UK Cohort Study. Clin Infect Dis. 2011; 53(2):107-13.

2. Gross $A E$, van Schooneveld TC, Olsen KM, Rupp ME, Bui TH, Forsung E et al. Epidemology and Predictors of Multidrug-Resistant Community-Acquired and Health Care-Associated Pneumonia. Antimicrob Agents Chemother. 2014; 58(9):262-8.

3. Ceccato A, González A, Heres M, Peluffo G, Monteverde A. Neumonía Asociada al Cuidado de la Salud. Medicina. 2014; 74:19-23.

14. Vallés J, Martin-Loeches I, Torres A, Díaz E, Seijas I, López MJ, et al. Epidemiology, antibiotic therapy and clinical outcomes of healthcare-associated pneumonia in critically ill patients: a Spanish cohort study. Intensive Care Med. 2014; 40(4):572-81.

5. Chen Jl, Slater LN, Kurdgelashvili G, Husain KO, Gentry CA. Outcomes of Health Care-Associated Pneumonia Empirically Treated with Guideline-Concordant Regimens versus Community-Acquired Pneumonia GuidelineConcordant Regimens for Patients Admitted to Acute Care Wards from Home. Ann Pharmacother. 2013; 47:919.

16. Attridge RT, Frei CR, Restrepo MI, Lawson KA, Ryan L, Pugh MJV, et al. Guideline-concordant therapy and outcomes 38:878-887.

7. Shorr AF, Zilberberg MD, Reichley R, Kan J, Hoban A, Hoffman J, et al. Validation of a Clinical Score for Assessing the Risk of Resistant Pathogens in Patients With Pneumonia Presenting to the Emergency Department. Clin Infect Dis. 2012; 54(2):193-198.

18. Aliberti S, Di Pasquale M, Zamboni AM, Cosentini R, Brambilla AM, Seghezzi S, et al. Stratifying Risk Factors for Mulidrug-Resistant Pathogens in Hospitalized Patients Coming From the Community With Pneumonía. Clin Infect Dis. 2012; 54(4):470-478.

19. Wang PH, Wang HC, Cheng SL, Chang $\mathrm{HT}$, Laio $\mathrm{CH}$. Selection of empirical antibiotics for health careassociated pneumonia via integration of pneumonia severity index and risk factors of drug-resistant pathogens. J Formos Med Assoc. 2016 May; 115(5):35663. 
20. Prina E, Ranzani OT, Polverino E, Cillóniz $C$, Ferrer $M$ Fernandez $L$, et al. Risk factors associated with potentially antibiotic-resistant pathogens in community-acquired pneumonia. Ann Am Thorac Soc. 2015; 12(2):153-60

21. Maruyama T, Fujisawa $T$, Okuno $M$, Toyoshima $H$ Tsutsui K, Maeda H, et al. A new strategy for healthcareassociated pneumonia: a 2-year prospective multicenter cohort study using risk factors for multidrug-resistant pathogens to select initial empiric therapy. Clin Infect Dis 2013; 57(10):1373-83

22. Brito V, Niederman MS. Healthcare-associated pneumonia is a heterogeneous disease, and all patients do not need the same broad-spectrum antibiotic therapy as complex nosocomial pneumonia. Curr Opin Infect Dis 2009; 22:316-25.

23. Carratalà J, Mykietiuk A, Fernández-Sabé N, Suárez C, Dorca J, Verdaguer R, et al. Health care-associated pneumonia requiring hospital admission: epidemiology, antibiotic therapy, and clinical outcomes. Arch Intern Med. 2007; 167(13):1393-9.

24. Webb BJ, Dascomb K, Stenehjem E, Dean N. Predicting risk of drug-resistant organisms in pneumonia: movin beyond the HCAP model. Respir Med. 2015 Jan; 109(1):1-
Health-care associated pneumonia:

review of published evidence

ABSTRACT

The concept of health-care-associated pneumonia (HCAP) arose from the presumption that the pathogens that cause the disease would be more likely to be multi-resistant microorganisms (MRMO) so the antibiotic treatment scheme micoor should be difeent (MT) som the acquired pneumonia(CAP). However, the evidence supporting this idea is not robust enough. Given the complexity of the topic, and its high impact on the exaggerated consumption of antibiotics, this review is presented. It is possible that the absence of risk factors usually described, or the presence of only one, suggests a low probability of MRMD, so the of only one, suggests a low probabily of MRM, so the therapeutic approach should be similar to that of CAP. On the contrary, in view of the accumulation of risk factors or in severe cases, MRMO coverage could be considered, through an extended spectrum scheme.

Key words: Health-care associated pneumonia - multidrugs microorganisms - pneumonia. 